\title{
Prediction of fatigue crack growth for semi-elliptical surface cracks using S-version fem under tension loading
}

\author{
M.S. Shaari ${ }^{1 *}$, M.R.M. Akramin ${ }^{2}$, A.K. Ariffin ${ }^{1}$, S. Abdullah ${ }^{1}$ and \\ Masanori Kikuchi ${ }^{3}$
}

\author{
${ }^{1}$ Department of Mechanical and Materials Engineering, \\ Faculty of Engineering \& Built Environment, \\ Universiti Kebangsaan Malaysia, 43600 Bangi, Malaysia \\ *Email: mohdshamil.shaari@yahoo.com \\ Phone: +60389216136 \\ ${ }^{2}$ Faculty of Mechanical Engineering, \\ Universiti Malaysia Pahang, 26600 Pekan, Pahang, Malaysia \\ ${ }^{3}$ Tokyo University of Science, 2641 Yamazaki, Noda, Chiba 287-8510, Japan.
}

\begin{abstract}
The finite element method (FEM) is commonly used is solving engineering problems including fatigue crack growth. The S-version FEM is an extended version of the FEM to predict fatigue crack growth. This method was developed into open-source software written in $\mathrm{C} / \mathrm{C}++$ language and built based on the UNIX operating system. The objective of this study is to perform a prediction for fatigue crack growth using the S-version FEM. This S-version FEM was structured using the global-local overlay technique that consisted of two separate meshes for global and local. Therefore, the computation process was solely focused on the local area instead of whole geometries. This undoubtedly reduced computation time and dependency on conventional FEM software. The geometry of the problem was modelled in FAST (pre-post) program and referred to as a global mesh, and the mesh was automatically generated in FAST. The location of the crack refers to the local mesh and at this stage smaller elements and finer mesh were made at the crack area. Local mesh was overlaid with global mesh. Then the global-local overlay approach was used to compute the engineering problem. The stress intensity factor (SIF) was calculated using the virtual crack closure-integral method (VCCM). The prediction of the fatigue crack growth results are shown in this study as well as the behaviour of the SIF corresponding to the crack growth. Analytical solutions were compared with the Sversion FEM to validate the results. A very good agreement was presented between the S-version FEM and analytical approach for the normalised SIF. In addition, regression analysis was performed to support the evidence. From the analysis, very small root mean square errors (RMSE) of 0.12 with high correlation coefficient $\left(R^{2}\right)$ of $99.74 \%$ were shown to confirm the findings. From this study, it can be concluded that the $\mathrm{S}$-version FEM is suitable to be used to predict fatigue crack growth for semi-elliptical surface cracks. The application of the S-version FEM benefits the user because using the overlay approach shortens the computation process.
\end{abstract}

Keywords: Fatigue crack growth (FCG), S-version Finite Element Method (S-version FEM), surface crack, virtual crack closure-integral method (VCCM). 


\section{INTRODUCTION}

There are many engineering structures such as those used in automotive components, aircraft manufacturing, bridges, buildings and more. Over the years, a series of improvements in technology, structural design and smart materials have been introduced to the public. However, disintegration of engineering structures has always posed a challenge for engineers to overcome. The deterioration phenomenon arises from cyclic stress further known as fatigue and should be detected before it reaches its threshold. Threshold occurs at stresses below the monotonic yield strength and for many years, researchers have conducted various tests to predict fatigue due to its relationship with the safety of human beings. Automotive components and vehicles are among the most important areas to focus as they are used to transport billions of people around the world. Therefore, it is important to provide sufficient data especially in fatigue for engineers to use in designing components, accordingly. The aluminum 7075-T6 is one of the most highly used materials for automotive components due to its high strength. This material is mainly used in automotive components including aircraft wing manufacturing $[1,2]$.

Numerical computation has been developed and used in various engineering fields since the last few decades. It is very common nowadays to use the finite element method (FEM) to analyse structures or components. Conventional software is able to produce results from structural analysis to fatigue life, based on scenarios or cases. The FEM is widely used in analysing nuclear power plants [3-5], vehicles in automation or aircrafts $[6,7]$ and for fatigue assessment [8-10]. However, existing FEM is still insufficient to analyse engineering problems due limitations related to complexity of problems and computation time [11-14]. Therefore, the S-version FEM was developed to enhance the existing FEM to overcome the constraints mentioned. The original concept of the Sversion FEM was introduced to the public by Fish [15] in 1992. The letter ' $S$ ' highlights the superimposition as local meshes are overlaid on global mesh. The local mesh is referred to as the problem(s), such as holes or cracks in the structure (global mesh). Hence, computation is focused on the local area instead of the whole domain as used in conventional FEM software. The concept and method for the S-version FEM will be duly discussed in the methodology section.

Scientifically, several approaches are used to validate works produced to verify findings from numerical computations, either through experimental results or analytical solutions. Newman and Raju are among the earliest researchers in the field of fatigue and fracture. In addition, several empirical formulations have been established related to finite plate since 1979. Surface crack was one of the works performed by them to attain fatigue crack growth in 1979. Their studies showed formulations of stress intensity factor (SIF) to predict the behaviour of semi-elliptical surface cracks [16]. Further researchers also confirmed that calculating the SIF is essential to indicate the distribution of crack growth along semi-elliptical surface cracks [17-21]. In general, the calculation of SIF is done using the virtual crack closure-integral method (VCCM) by computing the energy release rate on nodal forces and displacements. It is known that both of these are results from the finite element method. The semi-elliptical surface cracks is widely accepted in the fracture mechanics field either from plates under tension [22], notched round bars under cyclic tension and bending [23], thick cylinders [24] or even for weld heat-affected zones [19]. The present work shown in this study is fundamental work for larger aspects. Since it is open-source software, it can be modified and used for various cases such as for multiple cracks interaction, embedded cracks, multiaxial fatigue and more. In this study, 
the S-version FEM is used to predict the crack growth of semi-elliptical surface cracks of aluminum 7075-T6 under tension loading.

\section{METHODS AND MATERIALS}

\section{Concept to Formulation of S-Version FEM}

The concept $\mathrm{S}$-version FEM is shown in Figure 1. In this study, the S-version FEM is used to simulate surface cracks for a single crack under tension loading. A much simpler definition of this S-version FEM is a superposition mesh or superimposed mesh. It consists of a global mesh (coarser mesh) and local mesh (finer mesh) and both are superimposed in order to reduce computation time without jeopardising accuracy. The global mesh, $\Omega^{G}$, is generated according to geometry before being overlaid by the local mesh, $\Omega^{L}$. The local mesh represents the corresponding shapes and sizes of the cracks. In order to validate the results of Newman and Raju (1979) in this study, the semi-elliptical crack is used and the details will be discussed in the next section. The boundary of each region, is represented by $\Gamma$, where the superimposed boundary is represented by $\Gamma^{G L}$, whilst the boundary of the displacement and force (traction) are represented by $\Gamma^{u}$ and $\Gamma^{t}$, respectively.

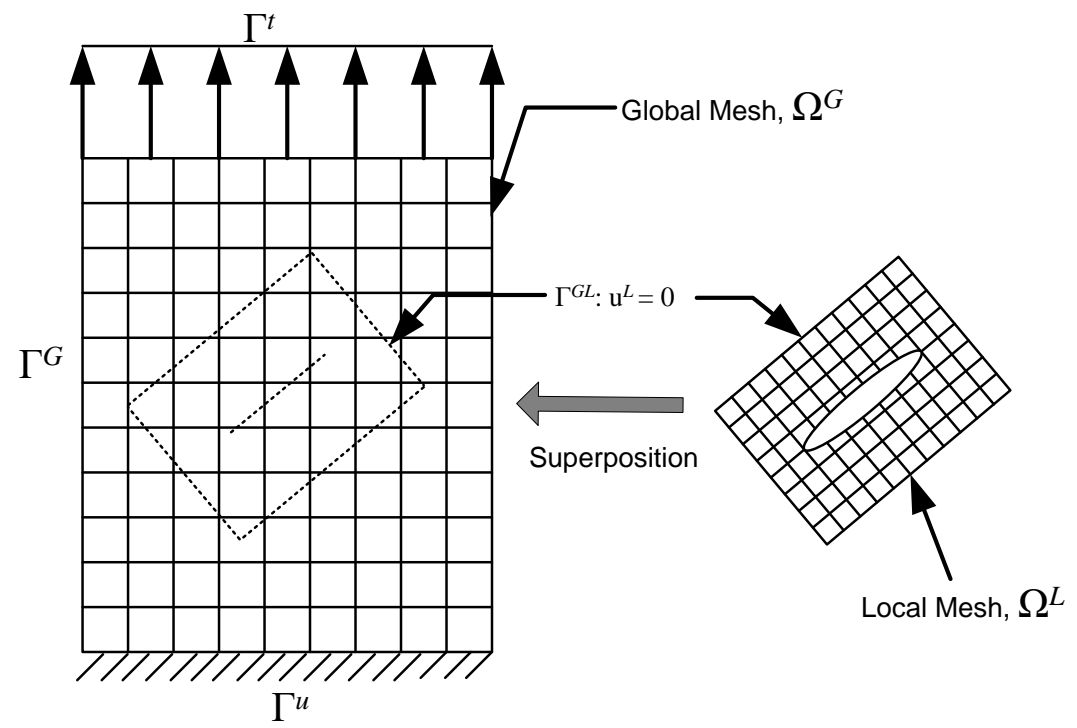

Figure 1. Concept of S-version FEM.

Therefore, the S-version FEM concept is now ready to be analysed. Each area (global and local) and, the displacement functions are defined independently. The displacement, $u$, for each are represented by $u^{G}$ and $u^{L}$ for the global area and local area, respectively. As shown in the previous figure, the displacement for the local area, $u_{i}^{L}$, is assumed to be zero to ensure continuity of the global area and local area boundaries. The equation below indicates the displacement functions for global and local:

$$
u_{i}=\left\{\begin{array}{cccc}
u_{i}{ }^{G} & i & \in & \Omega^{G}-\Omega^{L} \\
u_{i}{ }^{G}+u_{i}{ }^{L} & i & \in & \Omega^{L} \\
u_{i}{ }^{L}=0 & i & \in & \Gamma^{G L}
\end{array}\right.
$$


From the virtual work of principle:

$$
\int_{\Omega} \delta u_{i, j} D_{i j k l} u_{k, l} d \Omega-\int_{\Omega} \delta u_{i, j} b_{i} d \Omega-\int_{\Gamma^{t}} \delta u_{i} t_{i} d \Gamma=0 \quad(2
$$

The displacement functions in Eq. (1) are then applied to the virtual work of principle in Eq. (2) and shown in the following equation:

$$
\begin{aligned}
& \int_{\Omega^{G}} \delta u_{i, j}^{G} D_{i j k l} u_{k, l}^{G} d \Omega+\int_{\Omega^{L}} \delta u_{i, j}^{G} D_{i j k l} u_{k, l}^{L} d \Omega= \\
& \int_{\Omega^{L}}^{\delta} \delta u_{i, j}^{L} D_{i j k l} u_{k, l}^{G} d \Omega+\int_{\Omega^{L}} \delta u_{i, j}^{L} D_{i j k l} u_{k, l}^{L} d \Omega= \\
& \int_{\Gamma^{t}}^{\delta} \delta u_{i}^{G} t_{i} d \Gamma^{t G}
\end{aligned}
$$

Thus, the final form of the S-version FEM is obtained in the form of the following matrix:

$$
\left[\begin{array}{cc}
K_{G G} & K_{G L} \\
K_{L G} & K_{L L}
\end{array}\right]\left\{\begin{array}{l}
u^{G} \\
u^{L}
\end{array}\right\}=\left\{\begin{array}{l}
F_{G} \\
F_{L}
\end{array}\right\}
$$

where

$$
\begin{aligned}
& {\left[K_{G G}\right]=\int_{\Omega^{G}}\left[B^{G}\right]^{T}[D]\left[B^{G}\right] d \Omega^{G}} \\
& {\left[K_{G L}\right]=\int_{\Omega^{L}}\left[B^{G}\right]^{T}[D]\left[B^{L}\right] d \Omega^{L}} \\
& {\left[K_{L G}\right]=\int_{\Omega^{L}}\left[B^{L}\right]^{T}[D]\left[B^{G}\right] d \Omega^{L}} \\
& {\left[K_{L L}\right]=\int_{\Omega^{L}}\left[B^{L}\right]^{T}[D]\left[B^{L}\right] d \Omega^{L}} \\
& \left\{F_{G}\right\}=\int_{\Omega^{G}} N^{G} b_{i} d \Omega+\int_{\Gamma^{G}} N^{G} f_{i} d \Gamma \\
& \left\{F_{L}\right\}=\int_{\Omega^{G}} N^{L} b_{i} d \Omega+\int_{\Gamma^{G}} N^{L} f_{i} d \Gamma
\end{aligned}
$$

The reader would notice that, $\left[K_{G L}\right]$ and $\left[K_{L G}\right]$ matrices are symmetric and represent the stiffness matrix of the superimposed area. Matrix $[D]$ represents the material and $[B]$ is the element strain-displacement matrix. The nodal forces for global and local are the $\left\{F_{G}\right\}$ and $\left\{F_{L}\right\}$, respectively. By using the final matrix of the S-version FEM in Eq. 4, the displacement can be calculated for each node simultaneously - for global and local. As the local mesh grows accordingly, the global mesh does not change since the only region of interest is within the boundary of the crack front. The re-meshing process will also not include the global mesh. Hence it achieves the purpose of the superposition technique which is to reduce computation time. The stress intensity factor (SIF) is calculated along with the expansion of the local mesh using the energy release rate formulation.

The SIF is mainly used in fracture mechanics to predict crack growth by computing the stress state at the crack tip. In order to obtain the SIF, the energy release 
rate must be calculated first by using several established methods. The virtual crack closure-integral method (VCCM) is one approach that computes energy release rate. The VCCM was developed by Rybicki [25] in 1977.

The energy release rate formula, $G$, is shown in the following equation:

$$
G=\frac{1}{2 h}\left\{\left(u_{\alpha}^{\text {Upper }}+u_{\alpha}^{\text {Lower }}\right)\left(f_{\alpha}\right)+\left(u_{\beta}^{\text {Upper }}+u_{\beta}^{\text {Lower }}\right)\left(f_{\beta}\right)\right\}
$$

where

$$
\begin{aligned}
u & =u^{G}+u^{L} \\
d u & =\left(u_{G}+u_{L}^{\text {Upper }}\right)-\left(u_{G}+u_{L}^{\text {Lower }}\right) \\
& =\left(u_{L}^{\text {Upper }}+u_{L}^{\text {Lower }}\right)
\end{aligned}
$$

Eq. (7) explains how to acquire the changes in the displacement by using the VCCM. Where the $\alpha$ and $\beta$ both represent nodal points for the forces, $f$, and displacement, $u$, near the crack tip. Figure 2 shows a detailed illustration of the VCCM and the meaning of upper and lower nodal forces and displacement.

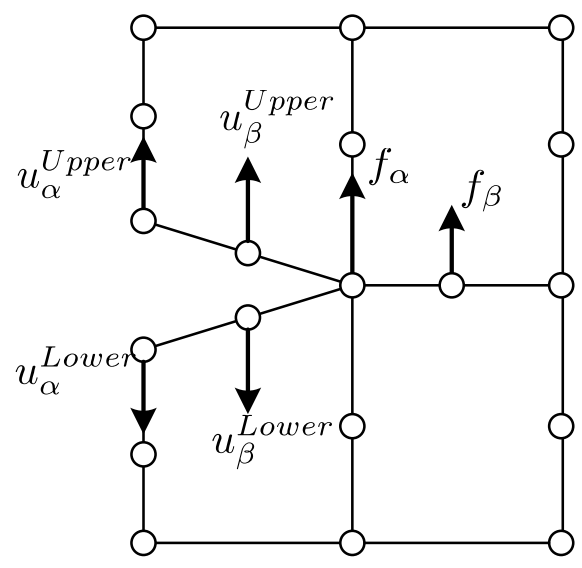

Figure 2. The concept of VCCM.

Once the energy release rate is obtained, the SIF can be computed by using the following equation:

$$
K_{I}=\sqrt{E G_{I}}, \quad K_{I I}=\sqrt{E G_{I I}}, \quad K_{I I I}=\sqrt{2 \mu G_{I I I}}
$$

where $K_{I}, K_{I I}$ and $K_{I I I}$ represent mode I, mode II and mode III type of loading, respectively. The widely known Paris' law is essential to predict fatigue crack growth rate. It is suitable to be used for $2 \mathrm{D}$ or $3 \mathrm{D}$ problems and the following equation shows Paris' law:

$$
\frac{d a}{d N}=C\left(\Delta K_{e q}\right)^{n}
$$

where, $d a$ is the crack growth rate and $N$ is the number of cycles. $C$ and $n$ constants are totally on material properties. 
Considerations of crack growth rate and crack growth direction are a must especially for mixed-mode loading cases. Even though it relates only for pure mode I loading condition in this study, crack growth direction and equivalent stress intensity factors, $\Delta K_{e q}$, should be taken into account. Richard et al. focused on the study of crack growth and developed a criterion for crack growth direction. Detailed explanations can be found in [26, 27]. Furthermore, the Richard criterion is integrated into the software to ensure that the S-version FEM is able to compute mixed-mode types of loading. Hence, the equivalent stress intensity factor equation is used in this study. The equivalent SIF, $\Delta K_{e q}$ based on Richard's criterion is expressed in the following:

$$
\Delta K_{e q}=\frac{\Delta K_{I}}{2}+\frac{1}{2} \sqrt{\Delta K_{I}^{2}+4\left(1.155 \Delta K_{I I}\right)^{2}+4\left(\Delta K_{I I I}\right)^{2}}
$$

where $\Delta K_{I,} \Delta K_{I I}$ and $\Delta K_{I I I}$, represent the range in stress intensity factors for mode I, mode II and mode III, respectively.

\section{Procedure for Crack Growth Simulation}

The procedure to predict fatigue crack growth using the numerical method has been widely used and accepted by researchers. The material properties of aluminum 7075-T6 consists of $71.7 \mathrm{GPa}$ for Young's modulus $(E), 0.33$ for poisson's ratio (v), $508 \mathrm{MPa}$ for yield and $980 \mathrm{MPa}$ for hardness. Figure 3 represents a superimposed mesh (global) where, a single crack (local) as shown in Figure 4 is overlaid on the finite plate. Both global and local meshes in Figure 3 and Figure 4 are modelled using the 20-node hexahedral element. The geometry of the plate is $200 \mathrm{~mm}$ by $200 \mathrm{~mm}$ with a thickness of $20 \mathrm{~mm}$. The local crack's length is $20 \mathrm{~mm}$ and its depth is $4 \mathrm{~mm}$. The reason for the selected geometry as well as the boundary conditions is to replicate the study by Newman (1979). The selected case study in this paper used $a / c$ of $0.4, c / b$ of $0.1, c / h$ of 0.1 and $a / t$ of 0.2 where $a$ is the crack depth, $c$ is the crack length, $b$ is the plate width, $h$ is the plate length and $t$ is the plate thickness. Boundary conditions were set to repeat the tension test and can be referred in Figure 5. The bottom of the plate is fixed in all directions and the upper side pulled with a uniform load of $10 \mathrm{MPa}$. Figure 6 shows a comparison between the plate before and after pressure is applied on the plate. Notice that there are slight differences to the displacement in the $y$ direction.

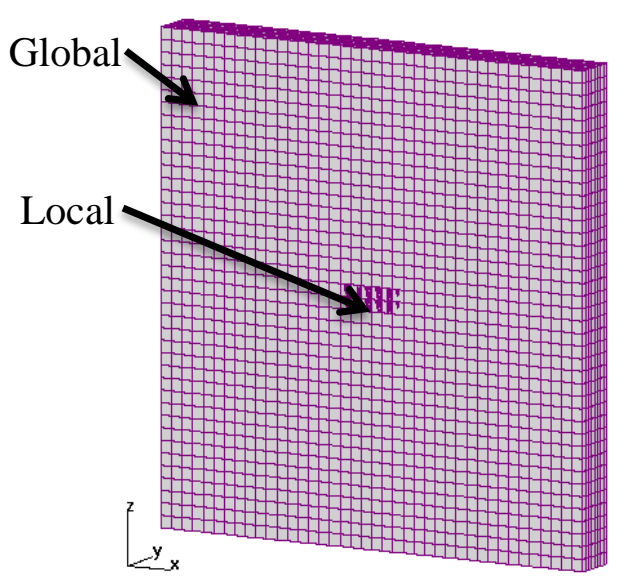

Figure 3. Geometry of plate combines with the crack. 


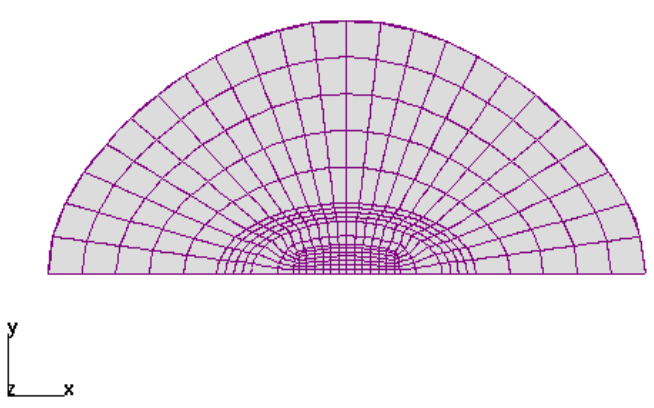

Figure 4. Local semi-elliptical surface crack

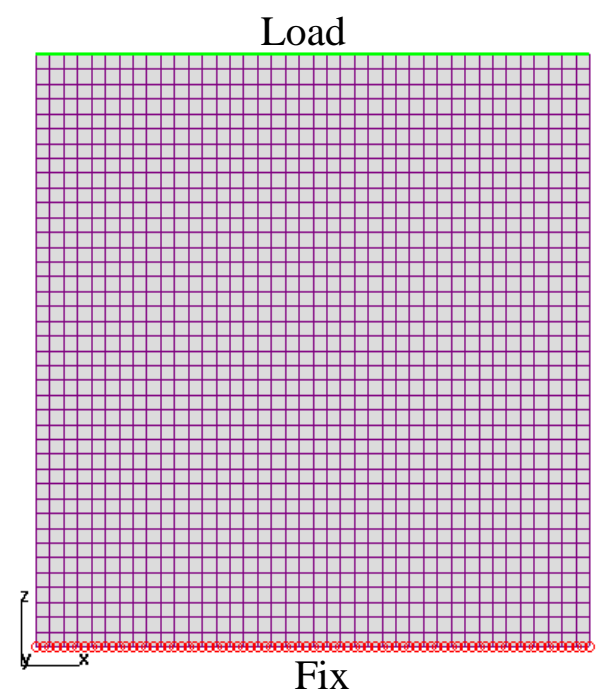

Figure 5. Global with boundary conditions

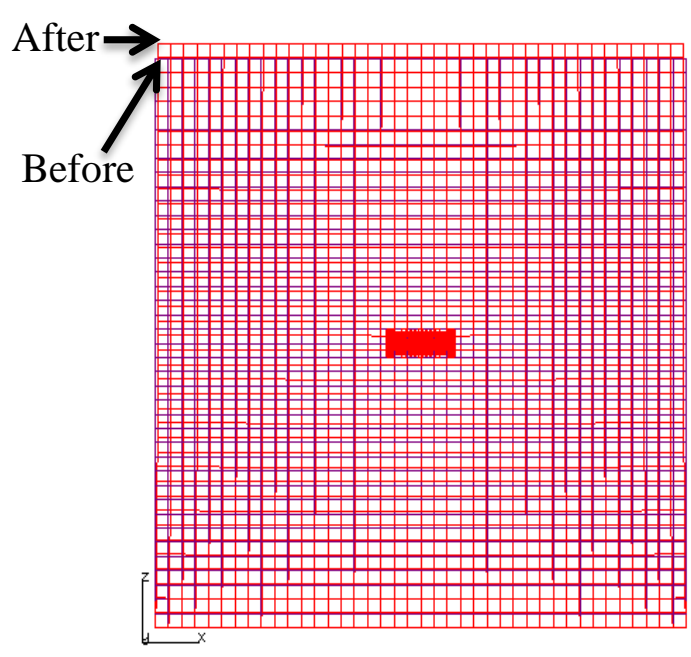

Figure 6. Displacement of global and local

\section{RESULTS AND DISCUSSION}

In this section, the S-version FEM results is evaluated in accordance with SIF, crack growth, and regression analysis. Figure 7 shows crack growth of a single crack on a finite plate corresponding to beach marks. The coordinates of the cracks can be referred to Figure 3 and Figure 4, respectively where crack propagation in the $y$ direction is the crack depth $(a)$ and the crack length $(c)$ propagates in the $x$ direction. For the crack length, the centre of the crack is noticeably at zero. Observe the crack depth $(a)$, it propagates but is immensely dissimilar to the crack length $(c)$ which only grows close to $2 \mathrm{~mm}$. The explanation for these phenomena is that the calculation of the equivalent stress intensity factors, $\Delta K_{e q}$, as in Figure 8 shows a steep slope for the crack length compared to the crack depth. In general, it is known that the SIF indicates crack growth and this happens for the crack length. By having a steeper slope of SIF, it gives an idea that the crack length experienced a stunted development. Normally, the fatigue crack growth rate is used to define Paris' $c$ value particularly from experimental results. However, the S-version FEM already considers the value initially set by users. Thus, the purpose of the fatigue crack 
growth rate graph in this study is to observe fatigue behaviour especially when comparing crack depth and crack length.

Figure 9 shows the behaviour of the SIF according to the beach marks along the crack front. Notice that the $x$-axis represents the degree of angle $(\phi)$ for the semi-elliptical surface cracks. From the results, because loading was applied uniformly to the plate, crack growth was of the same direction of the tension without having any concentrated phenomenon. As mentioned previously, the SIF correlates with the crack tendency to grow. As a matter of fact, the value of the SIF at $0^{\circ}$ and $180^{\circ}$, are relatively smaller than any other position on the crack front. This is due to sharper and tighter corner (semielliptical) shapes, where smaller values are obtained. It is very difficult for cracks to propagate due to insufficient stress. However, the highest value of SIF is shown at $90^{\circ}$ along the crack front. By having a sufficient value of stresses, cracks are easily propagated and this supports previous crack growth results.

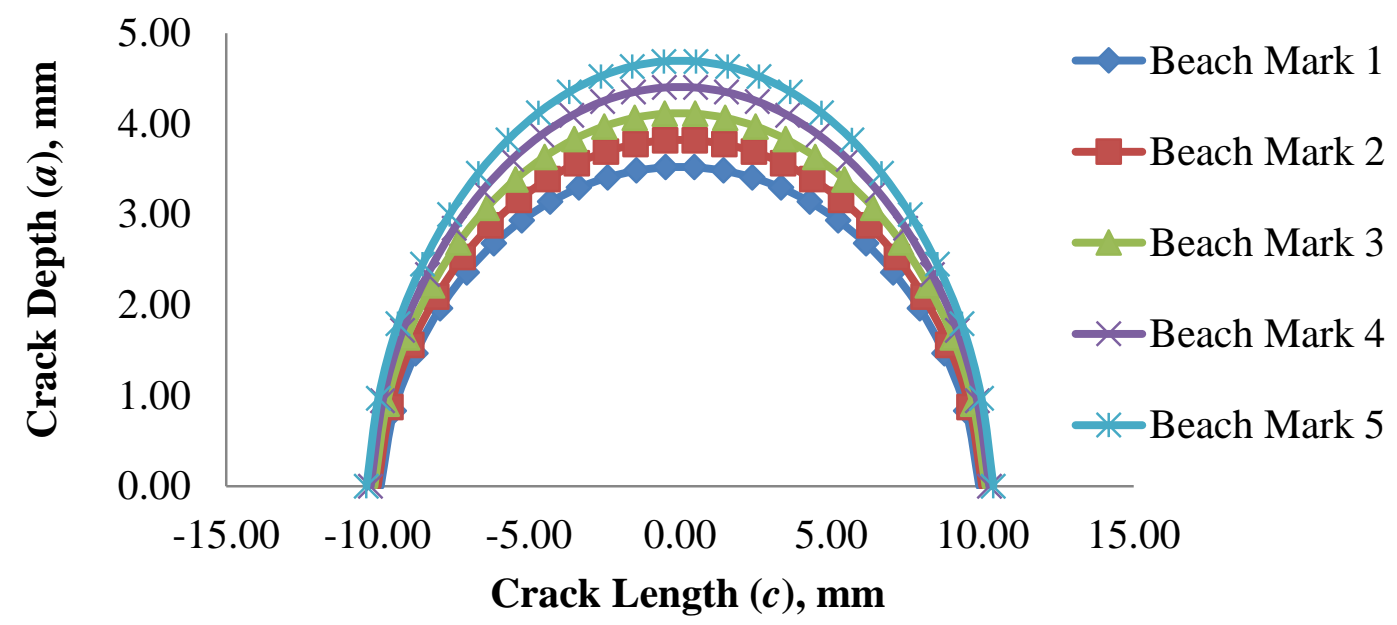

Figure 7. Prediction of fatigue crack growth for semi-elliptical surface cracks.

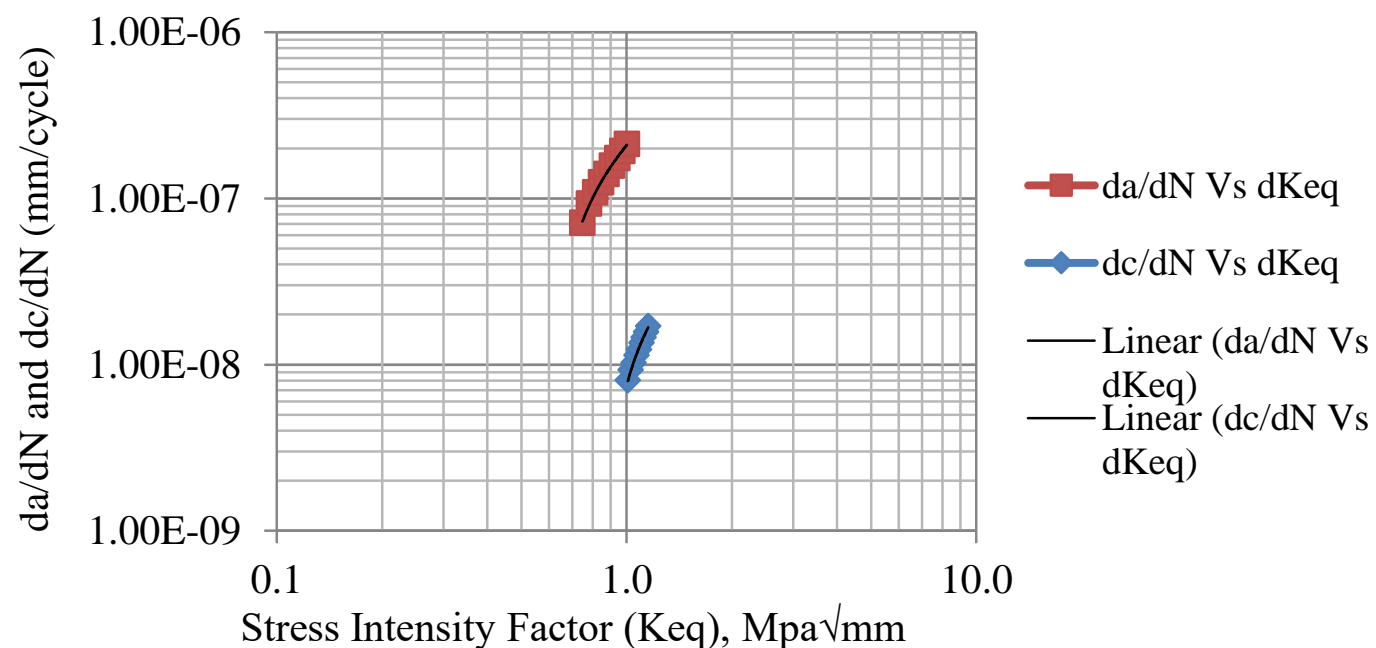

Figure 8. Comparison of fatigue crack growth behaviour between crack length $(c)$ and crack depth $(a)$. 
Based on the crack growth results, the crack depth and crack length grew proportionally with the number of cycles as shown in Figure 10. In addition, the crack length encountered a slight growth compared to the crack depth. The most essential result in this study is the normalised SIF of the S-version FEM compared to the analytical solution presented in Figure 11. The closed form empirical solution presented by Newman and Raju [12] for tension loading was followed closely in this study. Eq. (12) shows the formulation to calculate the SIF when tension loading was applied to the finite plate. Uniform loading is represented by $\sigma$ while $Q$ and $F$ are defined as shape factor and stressintensity boundary-correction factor, respectively. The shape factor, $Q$ is influenced by the crack's aspect ratio. Eq. (12) shows the formulation and differentiation between aspect ratios.

$$
K_{I}=\sigma \sqrt{\pi \frac{a}{Q} F}
$$

where, $Q=1+1.464\left(\frac{a}{c}\right)^{1.65}$ for $a / c \leq 1$;

$$
Q=1+1.464\left(\frac{c}{a}\right)^{1.65} \quad \text { for } a / c>1
$$

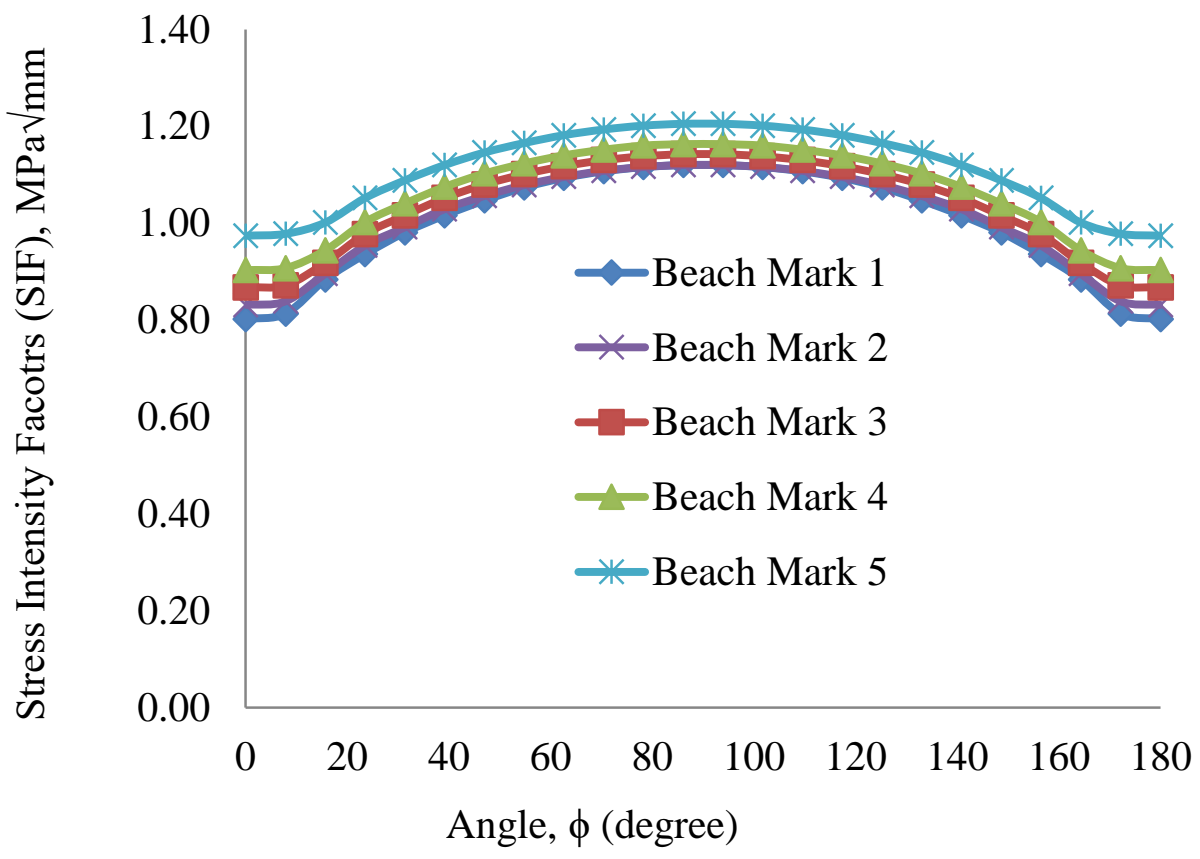

Figure 9. The behaviour of SIF according to beach marks and degree of angle.

Finally, the normalised SIF is computed from this normalise formula, $K_{I} / \sigma \sqrt{\pi(a / Q)}$ and plotted corresponding to the normalised angular direction as presented by Newman and Raju [16]. From Figure 11, the graph shows a very good agreement between both methods and to ensure accuracy of the validation, correlation analysis is performed later in this study. Figure 12 is the results of the regression analysis and the correlation coefficient $\left(R^{2}\right)$ obtained, showing a $99.74 \%$ correlation between the $S$-version FEM and analytical solution. Thus, the root mean square error (RMSE) is then calculated to support the evidence. Values from the RMSE calculation show a very small error of 0.12 . 


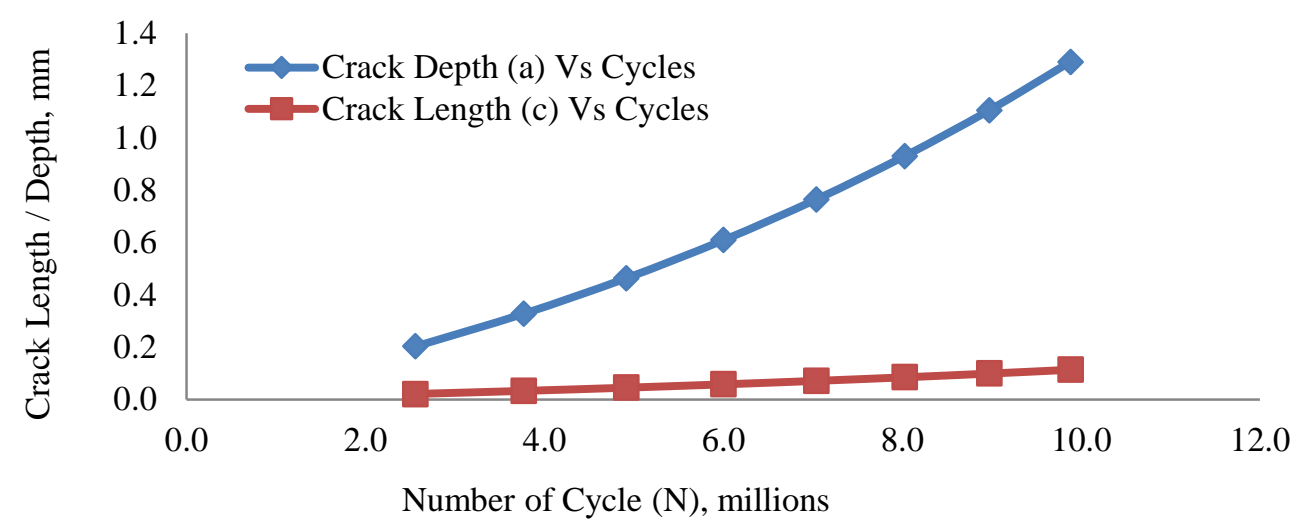

Figure 10. Comparison of crack length $(c)$ and crack depth $(a)$ behaviour according to fatigue cycles.

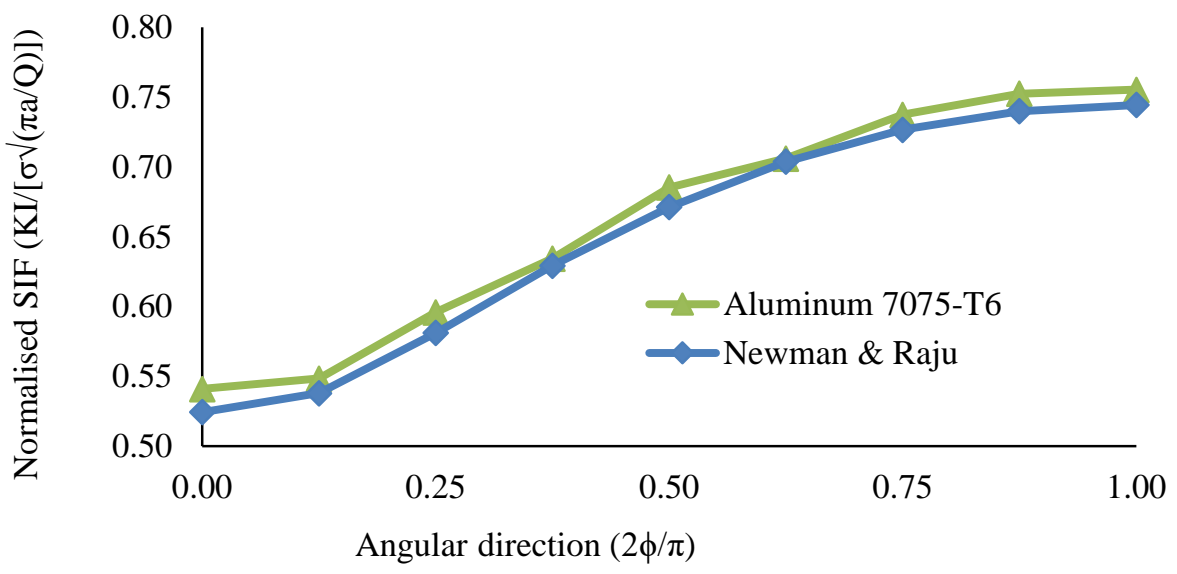

Figure 11. Comparison of normalised SIF of semi-elliptical surface cracks in a plate under tension between the S-version FEM and Newman and Raju's [12].

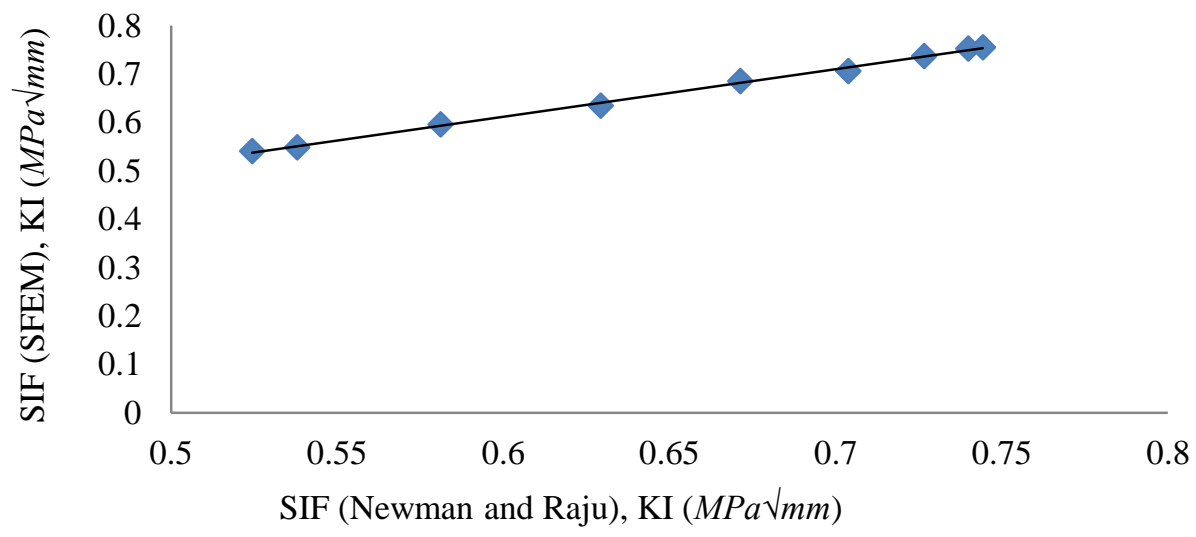

Figure 12. Regression analysis for stress intensity factors using the S-version FEM and Newman and Raju's [12].

\section{CONCLUSIONS}

The computational S-version FEM integrated with VCCM is presented to predict fatigue crack growth behaviour. This study adopted the superimposed FEM theory and used automatically regenerated meshing. This reduces computational time and memory usage. 
The prediction of fatigue crack growth for semi-elliptical surface cracks using S-version FEM was successfully performed under tension loading and the fatigue crack growth results were compared with the SIF, crack length and number of cycles. This paper also presented an important finding from the validation of normalised SIF. The results showed a good agreement between the S-version FEM and previously published literature. Finally, correlation analysis was performed to ensure accuracy of the results. From the analysis, the RMSE showed a very significant value of 0.12 , which was relatively low. The work presented in this study is considered as initial work to many other challenging and difficult problems especially relating to fatigue crack growth.

\section{ACKNOWLEDGEMENTS}

The author would like to acknowledge the Universiti Kebangsaan Malaysia (UKM), especially the laboratory of computational \& experimental mechanics (CEM) for allowing the research to be conducted using the high performance computer (HPC). This research is supported by the Ministry of Higher Education under Fundamental Research Grant Scheme. FRGS/2/2013/TK01/UKM/02/5.

\section{REFERENCES}

[1] Newman Jr JC, Anagnostou EL, Rusk D. Fatigue and crack-growth analyses on 7075-T651 aluminum alloy coupons under constant- and variable-amplitude loading. International Journal of Fatigue. 2014;62:133-43.

[2] Černý I, Sís J, Mikulová D. Short fatigue crack growth in an aircraft Al-alloy of a 7075 type after shot peening. Surface and Coatings Technology. 2014;243:20-7.

[3] Kikuchi M, Wada Y, Suga K. Surface crack growth simulation under mixed mode cyclic loading condition. Procedia Engineering. 2011;10:427-32.

[4] Okada H, Kawai H, Tokuda T, Fukui Y. Fully automated mixed mode crack propagation analyses based on tetrahedral finite element and VCCM (virtual crack closure-integral method). International Journal of Fatigue. 2013;50:33-9.

[5] Wu SY, Tsai BJ, Chen JJ. Elastic-plastic finite element analyses for reducers with constant-depth internal circumferential surface cracks. International Journal of Pressure Vessels and Piping. 2015;131:10-4.

[6] Dündar H, Ayhan AO. Non-planar crack growth analyses of multiple cracks in thin-walled structures. International Journal of Fatigue. 2016; 92(2): 596-604.

[7] Sivananth V, S.Vijayarangan. Fatigue Life Analysis and Optimization of A Passenger Car Steering Knuckle Under Operating Conditions. International Journal of Automotive and Mechanical Engineering. 2015;11:2417-29.

[8] Kamal M, Rahman MM. Finite Element-Based Fatigue Behaviour of Springs in Automobile Suspension. International Journal of Automotive and Mechanical Engineering. 2014;10:1910-9.

[9] Kamal M, Rahman MM. Fatigue Life Estimation Based On Continuum Mechanics Theory with Application of Genetic Algorithm. International Journal of Automotive and Mechanical Engineering. 2015;11:2586-98.

[10] Almaraz GMD, Tapia MG, Silva EET, Calderón EC. Fatigue Life Prediction Based On Macroscopic Plastic Zone on Fracture Surface of AISI-SAE 1018 Steel. International Journal of Automotive and Mechanical Engineering. 2010;1:29-37. 
[11] Kamal M, Rahman MM. Fatigue life estimation based on continuum mechanics theory with application of genetic algorithm. International Journal of Automotive and Mechanical Engineering. 2015;11:2686-98.

[12] Daud R, Ariffin AK, Abdullah S. Validation of crack interaction limit model for parallel edge cracks using two-dimensional finite element analysis. International Journal of Automotive and Mechanical Engineering. 2013;7:993-1004.

[13] Ahmad MIM, Arifin A, Abdullah S. Evaluating effect of magnetic flux leakage signals on fatigue crack growth of mild steel. Journal of Mechanical Engineering and Sciences. 2016;10:1827-34.

[14] Ahmad MIM, Arifin A, Abdullah S. Evaluation of magnetic flux leakage signals on fatigue crack growth of mild steel. Journal of Mechanical Engineering and Sciences. 2015;9:1727-33.

[15] Fish J. The s-version of the finite element method. Computers \& Structures. 1992;43:539-47.

[16] Newman Jr JC, Raju IS. Analyses of surface cracks in finite plates under tension or bending loads. NASA Technical Paper 1578. 1979.

[17] Kikuchi M, Wada Y, Shintaku Y, Suga K, Li Y. Fatigue crack growth simulation in heterogeneous material using s-version FEM. International Journal of Fatigue. 2014;58:47-55.

[18] Wada Y, Kikuchi M, Yamada S, Serizawa R, Li Y. Fatigue growth of internal flaw: Simulation of subsurface crack penetration to the surface of the structure. Engineering Fracture Mechanics. 2014;123:100-15.

[19] Kikuchi M, Wada Y, Shimizu Y, Li Y. Crack growth analysis in a weld-heataffected zone using S-version FEM. International Journal of Pressure Vessels and Piping. 2012;90-91:2-8.

[20] Shen W, Yan R, Liu E, Xu L. Fatigue growth behavior for surface crack in welding joints under combined compressive and bending stresses. International Journal of Fatigue. 2015;77:50-63.

[21] Nagai M, Miura N, Shiratori M. Stress intensity factor solution for a surface crack with high aspect ratio subjected to an arbitrary stress distribution using the influence function method. International Journal of Pressure Vessels and Piping.

[22] Lei Y. J-integral and limit load analysis of semi-elliptical surface cracks in plates under tension. International Journal of Pressure Vessels and Piping. 2004;81:2130.

[23] Carpinteri A, Brighenti R, Vantadori S. Surface cracks in notched round bars under cyclic tension and bending. International Journal of Fatigue. 2006;28:25160.

[24] Seifi R. Stress intensity factors for internal surface cracks in autofrettaged functionally graded thick cylinders using weight function method. Theoretical and Applied Fracture Mechanics. 2015;75:113-23.

[25] Rybicki EF, Kanninen MF. A finite element calculation of stress intensity factors by a modified crack closure integral. Engineering Fracture Mechanics. 1977;9:931-8.

[26] Richard HA, Sander M, Fulland M, Kullmer G. Development of fatigue crack growth in real structures. Engineering Fracture Mechanics. 2008;75:331-40.

[27] Richard HA, Fulland M, Sander M. Theoretical crack path prediction. Fatigue \& Fracture of Engineering Materials \& Structures. 2005;28:3-12. 